\title{
Continuous Abelian Sandpile Model in Two Dimensional Lattice
}

\author{
N. Azimi-Tafreshi, E. Lotfi, S. Moghimi-Araghi* \\ Department of Physics, Sharif University of Technology, \\ Tehran, P.O.Box: 11365-9161, Iran
}

\begin{abstract}
We define a new version of sandpile model which is very similar to Abelian Sandpile Model (ASM), but the height variables are continuous ones. With the toppling rule we define in our model, we show that the model can be mapped to ASM, so the general properties of the two models are identical. Yet the new model allows us to investigate some problems such as effect of very small mass on the height probabilities, different boundary conditions, etc.

PACS: 64.60.av, 45.70.Cc, $05.65+\mathrm{b}$,

Keywords: Self-Organized Criticality, Sandpile models.
\end{abstract}

\section{Introduction}

The concept of self-organized criticality (SOC) was first introduced by Bak, Tang and Wiesenfeld [1] through a simple model. This concept is believed to be the underlying reason for the scaling laws seen in a number of natural phenomena [2]. Their model, which was called Abelian Sandpil Model (ASM) is still the simplest, most studied model of SOC, in which many analytical results has been derived. For a good review see ref. [3].

Many exact results are derived in this theory. The first analytical calculation, which paved the road for other analytical results, was done by Dhar [4]. In his paper, Dhar computed the number of recurrent configuration and showed that all occur with equal probability. Later, in [5], Majumdar and Dhar calculated the probabilities of occurrence of some specific clusters, known as Weakly Allowed Clusters (WAC's). The simplest of these clusters is one-site height one cluster. The probabilities of other one-site clusters with height above 1 was computed in ref. [6]. There are many other analytical results, among them one can mention the results on boundary correlations of height variables and effect of boundary conditions [7, 8, 9, 10, 11, 12, on presence of dissipation in the model [9, 10, 13], on field theoretical approaches [17, 9, 14, 15, 18], on finite size corrections [11, 5] and many other results [3].

In ASM, the height variables take only integer values. There are some other models that in which the height variables can be real [19, 20. In Zhang model, when a site topples, all of its energy (height) is distributed among it neighbors and nothing is left for the original site. The dynamics

*e-mail: samanimi@sharif.edu 
does not have the Abelian property that the ordinary sandpile model has. Therefore, few analytical results are derived in this model [21]. On the other hand the model introduced by Gabrielov, called Abelian Avalanche Model (AA Model), is Abelian and more or less resembles the original ASM. The model is defined on a general graph and many general properties are investigated. In this paper we introduce a model similar AA model, defined on a square lattice and investigate some more specific properties. This model, in which the height variables can adopt any (positive) real numbers, allows us to investigate some yet unanswered question in the original ASM, such as the effect of dissipation on all height probabilities.

In this paper we first introduce our model, and show that it can be mapped to the usual ASM. Using analytical results and simulations, we derive some properties of the model. Next we introduce dissipation to the model and see what will be its effect probabilities of height variables. Then we investigate the model in presence of some boundary conditions.

\section{Continuous Abelian Sandpile Model}

Consider a $L \times L$ square lattice. To each site $u=(i, j)$ a continuous height variable, $h(u)$ is assigned, where without loss of generality we assume these variables are in $[0,1)$. The evolution rules consist of the following rules: 1) At each time step, a site is selected randomly and an amount of sand is added to it and other height variables are unchanged. The amount of sand added to the site is a random real number in the interval $[p, q] \in[0,1)$. The distribution function could be any function, but for simplicity we take it to be uniform on the interval $[p, q]$. If height remains below one, the new configuration is stable and we go to the next time step. 2) If height of the sand at that site becomes equal to or greater than one, the site becomes unstable and topples in the following way: it gives an amount of sand with height $1 / 4$ to either of its neighbors. In other words $h(v) \rightarrow h(u)-\Delta^{C}(u, v)$ for all $v$, where $\Delta^{C}(u, v)$ is the toppling matrix of the CASM and is defined as

$$
\Delta^{C}(u, v)\left\{\begin{array}{cc}
1 & u=v \\
-1 / 4 & |u-v|=1 \\
0 & \text { otherwise }
\end{array}\right.
$$

So if the height of a site is $1+\epsilon$, after toppling its height will be $\epsilon$ and the heights of neighbors will be added by the amount $1 / 4$. As a result of this toppling, some of the neighbors may become unstable and an avalanche may occur. Note that in the Zhang model, all the energy (height) of the site is distributed to the neighbors and the site keeps no energy.

It is clear that the model, which we call it Continuous Abelian Sandpile Model (CASM), has the same sprite as of ASM. We show in this paper that this model can be mapped to ASM and hence has the very same property of it; that is, it shows self-organized criticality and some of its properties could be found analytically. This model has some similarities to the one introduced in [22] and [23]. In the paper by Ghaffare et al. , the problem is investigated using mean field approach and the distribution of avalanche sizes is discussed using simulation. In the paper by Tsuchia and Katori, the height variables are still integer numbers, but if we take a large number for the threshold value, 


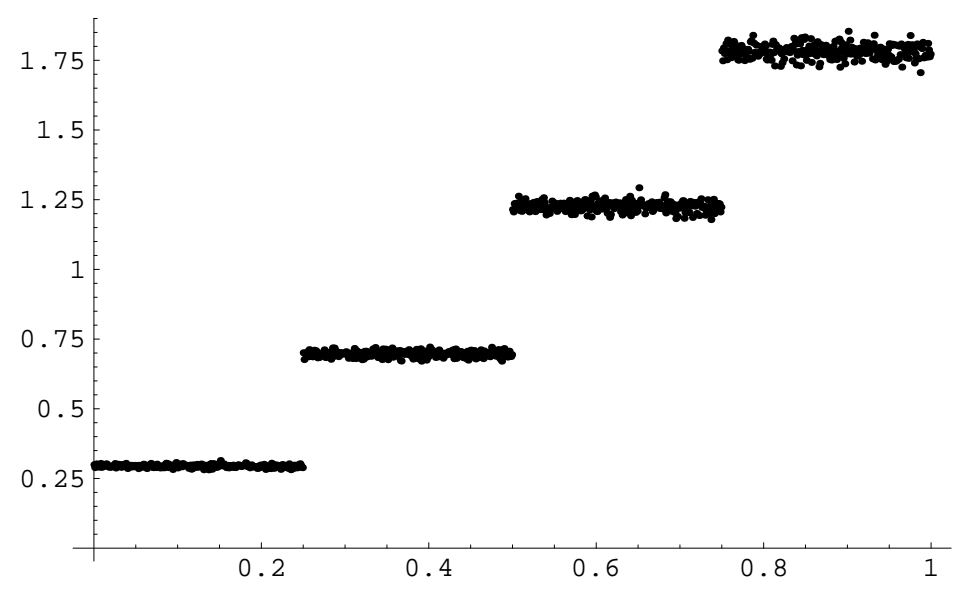

Figure 1: The probability density profile of height variables

it will approach to our model. They have considered the avalanche size distribution and correlation functions when dissipation is present in their model. Here we investigate the model at and off criticality and consider some boundary conditions and some other variants of the model.

A simulation is done and the probability distribution is found to be like Fig. 1. The result is more or less the same as the one obtained in [24]. As you see, the probability distribution has four plateaus, each have a length equal to $1 / 4$, but their heights increase step by step. It is easy to find the probability of finding a site at each of these plateaus. Labeling the plateaus by numbers 1,2 , 3 and 4 , it is found that $p(1)=0.0736 \pm 0.00008, p(2)=0.1741 \pm 0.0004, p(3)=0.3064 \pm 0.0007$, $p(4)=0.4456 \pm 0.001$, where $p(k)$ is the probability of being in $k$-th plateau. These probabilities are just the same as the ones in ASM with $p(k)$ being the probability of finding a site with height $k$. This brings to mind that there should be a close relation between the two models. In this section we prove two propositions:

Proposition 1: There is a (many to one) mapping from configurations in CASM to configurations in ASM, which preserves the dynamics. That is, it does not matter that you first apply the dynamics in CASM and then map it to ASM or vice versa.

Proposition 2: The probability distribution is piecewise constant.

To prove proposition 1 , we define the map $\mathcal{M}$ from configuration space of CASM $(\mathcal{C})$ to configuration space of $\operatorname{ASM}(\mathcal{A})$. Take $c \in \mathcal{C}$ and $a \in \mathcal{A}$. Let's call the height variable at site $u$ in configurations $c$ and $a, h_{u}^{C}$ and $h_{u}^{A}$. The action of $\mathcal{M}$ on $c$ is $a$ if

$$
h_{u}^{A}=f\left(h_{u}^{C}\right),
$$

where

$$
f(x)=[4 x]+1 .
$$

This means that the $k$-th $1 / 4$ goes to $k$.

Now it is easy to see that the map $\mathcal{M}$ preserves the dynamics of the theory. The first rule of evolution is to add sand. If the value of added sand in CASM takes the height of a site to $n$ higher 


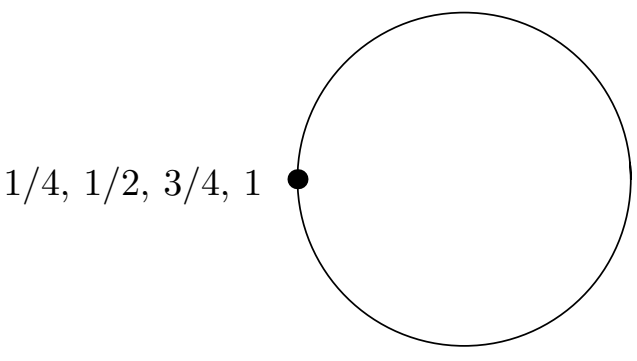

(a)

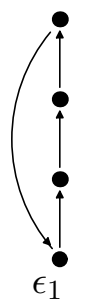

(b)

Figure 2: (a) The aggregate probability could be defined of a circle with perimeter $1 / 4$. (b) The effect of toppling on relative height probabilities for different $\epsilon$ 's.

plateau, it is equivalent to adding $n$ sand grains to the site. Although it does not matter, we may assume $q \leq 1 / 4$ so that at each time step, at most one sand grain is added to the site. The other evolution rule is the toppling rule. Calling $T_{u}^{C}$ and $T_{u}^{A}$ the toppling operators that only topple the site $u$ in CASM and ASM respectively, we would like to show if $a=\mathcal{M} c$ then $T_{u}^{A} a=\mathcal{M} T_{u}^{C} c$, that is the toppling rule and the mapping commute, hence the map preserves the dynamic. We should just note that the toppling matrix of ASM, $\Delta^{A}$, is 4 times the toppling matrix of CASM and all of its elements are integer. So the height variable of the configuration $\mathcal{M} T_{u}^{C} c$ at site $v$ will be $\left[4 h_{v}^{C}-\Delta_{u, v}^{C}\right]+1=\left[4 h_{v}^{C}\right]+1-\Delta_{u, v}^{A}=h_{v}^{A}-\Delta_{u, v}^{A}$ which is the height variable of $T_{u}^{A} a$ at the same site. So the first proposition is proved.

The proof of second proposition is a bit lengthier. To begin, we define the probability distribution function $P(h) d h$ which is the probability of finding a site with height in the interval $[h, h+d h]$. Also we define the aggregate probability distribution function as

$$
P_{I}(\epsilon)=P(\epsilon)+P\left(\frac{1}{4}+\epsilon\right)+P\left(\frac{1}{2}+\epsilon\right)+P\left(\frac{3}{4}+\epsilon\right)+P(1+\epsilon),
$$

where $0 \leq \epsilon<1 / 4$. Note that although in the stable states we have $P(1+\epsilon)=0$, but we have added such term in this definition. It is easy to prove that the toppling process does not change the aggregate probability function, since in a toppling process, the height of a site either increases by the amount $1 / 4$ or decreases by the amount 1 , that is if it has been in the set $\{\epsilon, 1 / 4+\epsilon, 1 / 2+$ $\epsilon, 3 / 4+\epsilon, 1+\epsilon\}$ it will remain in the same set. So the profile of the aggregate probability function is not altered under toppling.

Now we consider the effect of the other evolution rule, the rule of adding sand, on the aggregate probability function. As you see we have somehow identified the heights $k / 4+\epsilon$ where $k$ is an integer. As a result we may consider the whole interval of possible heights as a circle with perimeter $1 / 4$ (see Fig. 2a). Adding height $\delta h$ to a site takes a point on this circle to a point which is away from it with the amount $\delta h$, no matter where the starting point is. This means that after sufficient time that the system reaches stationary state the probability function on the circle is uniform, that is the aggregate probability function is uniform.

In the end we have to show that the relative probabilities of $p(k / 4 \epsilon)$ are independent of $\epsilon$. The toppling process may alter these relative probabilities. As shown in Fig. 2b, the effect of the toppling process is the same for different $\epsilon$ 's. This is due to the fact that the transition probabilities 
are independent of $\epsilon$, as all of them are mapped to the same configuration in ASM. Therefore the relative probabilities will be the same if we let the system evolve under many topplings and reach stationary state. This completes the proof of the second proposition.

From these two propositions, one can determine the density in the configuration in the stationary state. Let's call the subset of all configurations in CASM that are mapped to the specific recurrent configuration $a$ in $\mathcal{A}$ by $C_{a}$. By proposition one, it is clear that the probability of being in $C_{a_{1}}$ is equal to the probability of being in $C_{a_{2}}$, where $a_{1}$ and $a_{2}$ are two recurrent configurations in ASM. By proposition two, one deduces that within a set like $C_{a}$, the probability distribution is uniform, so it is easily seen that in steady state he density in the configuration state is uniformly distributed in the recurrent subspace.

All of this could be seen in through a naive reasoning. Consider the same square lattice, but assume that there are $N / 4$ connections between each two neighbors. Through any connection, only $\delta=1 / N$ grain of sand can be transferred. This means that when a toppling occurs, the height of neighbors is increased by the amount $1 / 4$. As you can easily see, if we take $N=4$, apart from a factor of 4 , we arrive at the usual ASM. However, if we take the limit of $N \rightarrow \infty$ we will have the CASM. It is easy to see that most of the properties of this model, are the same. Also neglecting dissipation, the model introduced in [23] is a special version of this model. In the next section we introduce some variants of the model that may have dissipation.

\section{Dissipative CASM}

In usual models, the dissipation parameter, which is usually called mass, can have only integer values, though some simulations with continuous height variables are done [22, 25] and using analytical results many aspects of massive ASM, with arbitrary continuous mass has been derived [17, 8]. To add dissipation to the model introduced in the previous section, one can increase the threshold height to $1+t$ instead of one, where $t$ is a positive real number. This means that if the height of the site $u$ becomes equal to or greater than $1+t$ then

$$
\begin{aligned}
h_{u} & \rightarrow h_{u}-1-t, \\
h_{v_{i}} & \rightarrow h_{v_{i}}+1 / 4,
\end{aligned}
$$

where the $v_{i}$ are the neighbors of the site $u$. As you see in a toppling process an amount of sand equal to $t$ is lost. One can see that this model resembles the usual ASM with dissipation: if we take $t=k / 4$, where $k$ is a positive integer number, then the mapping introduced in previous section, takes this CASM to an ASM which dissipates $k$ sand grains in each toppling. Simulation results show that still we have the same plateaus, of course with a new one for the sites with height greater than one. As we increase the dissipation, the distances between the plateaus' heights becomes

less, at $t=0.4$ the two last plateaus could not be easily distinguished. (Fig 3.) The probability distribution function for the cases $t=0.05,0.1,0.2,0.4$ is shown in Fig. 3.

The other interesting data we can investigate in this model is the behavior of $p(k)$ 's as functions of mass parameter. This has been done for some WAC's in [17] in an analytical way for arbitrary 


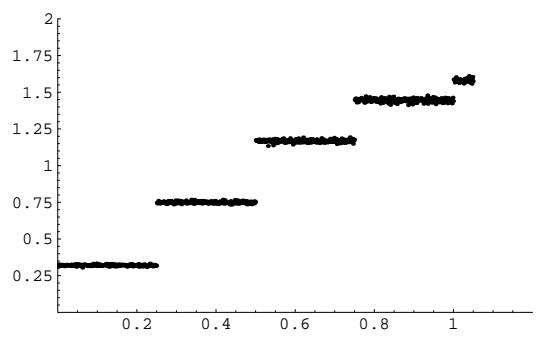

(a)

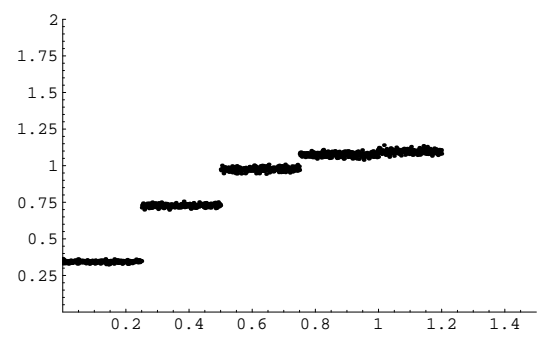

(c)

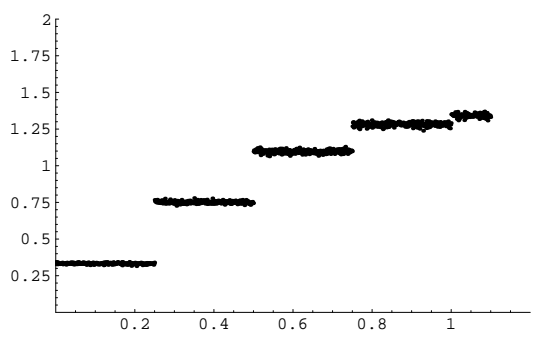

(b)

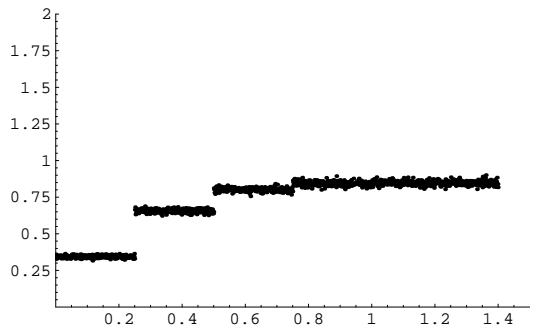

(d)

Figure 3: The probability distributions profile for mass different mass parameters. The mass parameters are $0.05,0.1,0.2$ and 0.4 for the graphs (a), (b), (c) and (d), respectively

value of $t$, though in the original model only positive integer values of $t$ are meaningful. Again in our model, the mass could take any positive real number. In Fig. 4 the probability of finding a site with with different heights is shown and in the case of height one, is compared with analytic results. The simulation result coincides with the antilytic one up to $t=0.25$, which corresponds to one grain of sand loss in ASM. After that, the two results are not in agreement, we were not been able to find the origin of this disagreement. Also it is observed that for probabilities of heights one and two and three, we have a maximum value that occurs in non vanishing mass parameter, however it seems that for height four the maximum probabilities happen at $t=0$ or at a value $t<0.0001$. The maximum values for probabilities of heights 1,2 and three occur at $t=0.24$, $t=0.08$ and $t=0.004$.

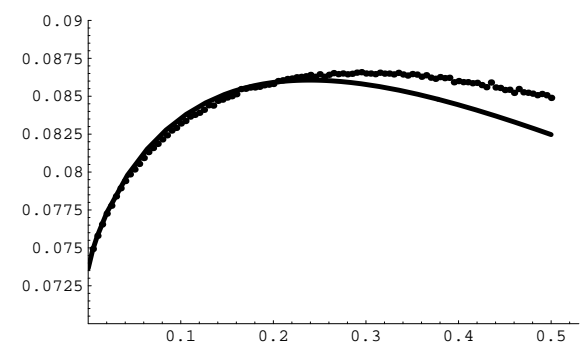

(a)

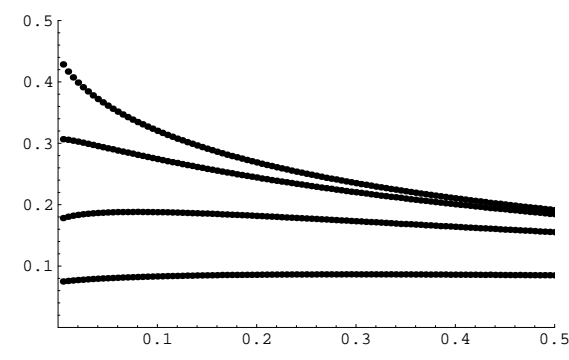

(b)

Figure 4: The probability of different heights as functions of mass: (a) The comparison between simulation and analytical results for probability of height one. (b) The probabilities of heights 1,2,3 and 4. 
It is also possible to investigate models where the mass parameter is not uniform, but is a smooth function of spacial coordinates. This may lead to some marvelous results if mass is considered as source of disorder in the theory.

\section{Some Boundary Conditions}

Two well-known boundary conditions (BC's)may be imposed along the boundaries of usual ASM: open and closed. A boundary site is open if in a toppling loses 4 grains, one of which falls off the boundary. It is closed if the number of grains it loses is equal to the number of its neighbors. Additionally, some other boundary conditions are investigated [12. Switching from one boundary condition to other at a specific point, is equivalent to introduce a boundary changing operator at the point in the field theoretic description of the model. These operators have been found to have weights equal to $-1 / 8$ [11, 12]. In our model, we are able to go from one boundary condition to the other in a smooth way. The open boundary site could be thought as a closed boundary site which has a mass parameter equal to one (in ASM language). Now we can introduce half-open boundary sites where their mass parameter is nighter zero nor one, but something in between. Although it is clear that under renormalization, mass parameter increases and therefore any small nonzero value of it will eventually have the same effect, but it is still interesting to examine this. Also we have the opportunity to alter other elements of the toppling matrix in a continuous way. To do this, we introduce a new boundary condition called reflective boundary condition. In the new boundary condition, not only we take the matrix elements $\Delta_{i i}$ be $3 / 4+t$ for the boundary sites, but we also assume that $\Delta_{i j}=-(1 / 4+t)$ for the link between the boundary sites and the sites who are just one row above the boundary. This means that there is no dissipation, as one can verify $\sum_{j} \Delta_{i j}=0$ for all the sites. There is an equivalent version of such boundary condition in ASM: if the height of a boundary site becomes more than four, then it topples, giving one sand grain to each of its neighbors who sit at the boundary line, and two sand grains to its other neighbor; that is, instead of throwing a sand grain away, it is reflected towards the system. The boundary condition we consider, is a continuous version of such condition.

Let's first concentrate on open and closed boundary conditions. It has been shown that operator effecting the change from closed to open, or from open to closed, is a boundary primary field of weight $-1 / 8$, belonging to a $c=-2$ logarithmic conformal field theory [11]. Such changes happen abruptly, the matrix elements $\Delta_{i i}$ corresponding to boundary sites change from 3 to 4 just at a single point. We would like to see if these changes are made smoother, what happens to the boundary operator. As an example one can take $\Delta_{i i}=3+\left(1+\tanh \left(\alpha\left(i-i_{0}\right)\right)\right) / 2$ where $\alpha$ is an arbitrary real number. Although by numerical results one can see that boundary operators have the same $-1 / 8$ scaling dimension, but one is also abe to derive some analytical results. Let's take the following boundary condition

$$
\Delta_{i i}=\left\{\begin{array}{cc}
1 & i<1 \text { or } i>n \\
3 / 4 & 1<i<n \\
3 / 4+1 / 8 & i=1 \text { and } i=n
\end{array}\right.
$$




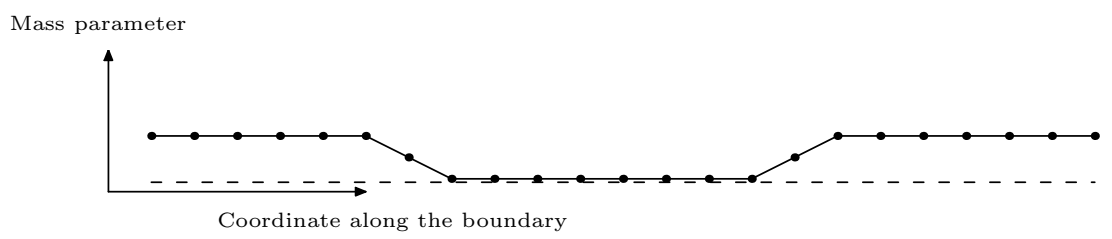

Figure 5: A non-sharp boundary condition: Two of the boundary sites are half-open

This means that the site in the interval $I_{0}=[2, n-1]$ are closed, the sites 1 and $n$ are half open and other boundary sites are open( See Fig. 5). The ratio of partition functions of theory with two different boundary condition (e.g. our BC and open BC) yields the two point correlation function of boundary changing operators. Following [11, 12] we define a defect matrix $B$ which in our case will be $1 / 4$ of identity matrix in the subspace of the sites in $I_{0}$, and has two other nonzero matrix elements $B_{11}=B_{n n}=1 / 8$. Therefore the ratio of the partition functions turns out to be

$$
\frac{Z^{\text {new }}}{Z^{o p}}=A e^{\frac{-2 G}{\pi}(n-1)} n^{1 / 4}\left(\left(1-\frac{2}{n}\right)^{\frac{1}{4}} e^{\frac{2 G}{\pi}}+2\left(1-\frac{1}{n}\right)^{\frac{1}{4}}+e^{\frac{-2 G}{\pi}}\right)
$$

Here $A$ is a constant and $G$ is the Catalon constant. As it is clear, for large $n$ 's, we will arrive at the results obtained in [11. The next leading terms behave as $n^{-3 / 4}$ that could be assigned to some operators having scaling dimension equal to $3 / 8$. Such fields do exist in $c=-2$ theory.

The other $\mathrm{BC}$ we investigate here is the reflecting BC. Let's take the boundary site $(i, 0)$ to be a reflecting one. Let $(i, 1)$ be the bulk site which is a neighbor of the site $(i, 0)$. As said before the reflecting $\mathrm{BC}$ implies that $\Delta_{(i, 0)(i, 0)}=3 / 4+t$ and $\Delta_{(i, 0)(i, 1)}=-1 / 4-t$. All other matrix elements are unchanged. Following [11] we take a segment of the boundary with length $n$ to be a reflecting $\mathrm{BC}$, while other sites are open/closed. The ratio $Z_{R} / Z_{o p / c l}$ will give us the two point function of boundary changing fields. Here $Z_{R}$ is the partition function of the model with the boundary condition expressed above and $Z_{o p / c l}$ is the partition function of the model if all the boundary sites are assumed to be open/closed.

All determinants have the Toeplitz form $\operatorname{det}\left(\sigma_{i-j}^{\alpha \beta}\right)$ where $\alpha$ and $\beta$ can take values 0 and 1 and determine whether we are considering the boundary site or the site just one row above the boundary. As the matrix elements $B_{\alpha=1, \beta}=\Delta_{\alpha=1, \beta}^{R}-\Delta_{\alpha=1, \beta}^{c l / o p}=0$, the determinants we are considering reduce to determinants of $n \times n$ matrices. The entries of these matrices are

$$
\sigma_{m}^{c l / o p}=\delta_{m, 0}+\frac{t-a}{4} G^{c l / o p}((0,1) ;(m, 1))-\frac{t}{4} G^{c l / o p}((0,2) ;(m, 1))
$$

where $a=0,1$ refer to the cases closed/open and $G^{c l / o p}$ is inverse of $\Delta^{o p / c l}$. By the method of images one finds that the coefficients $\sigma_{m}$ are the Fourier coefficients of a function $\tilde{\sigma}$,

$$
\begin{aligned}
\tilde{\sigma}^{o p}(k)= & (2-2 \cos (k))^{\frac{1}{2}} \\
& \left((2 t(\cos (k)-3)+(t-1)) \sqrt{\frac{1-\cos (k)}{2}}+(2 t(2-\cos (k))-(t-1)) \sqrt{\frac{3-\cos (k)}{2}}\right) \\
\tilde{\sigma}^{c l}(k)= & (1+t)+t(2-2 \cos (k))^{\frac{1}{2}}\left(\sqrt{\frac{1-\cos (k)}{2}}-\sqrt{\frac{3-\cos (k)}{2}}\right)
\end{aligned}
$$


The asymptotic value of Toeplitz determinant is given by using a theorem due to Widom [26]. Using this theorem one finds $\left\langle\phi_{(o p, R)} \phi_{(R, o p)}(n)\right\rangle \sim n^{1 / 4}$ and $\left\langle\phi_{(c l, R)} \phi_{(R, c l)}(n)\right\rangle \sim n^{0}$. Therefore the scaling exponents of the $\mathrm{BC}$ changing operators $\phi_{(o p, R)}$ and $\phi_{(c l, R)}$ are $-1 / 8$ and zero respectively; that is, the reflecting $\mathrm{BC}$ is more or less the same as closed $\mathrm{BC}$, which could be expected by hand-waving arguments.

At the end we would like to say that some other bulk models could be developed when the elements of toppling matrix $\Delta$ could be real values: one can add some spacial asymmetries or add ellipticity to the problem. This issue is under consideration [27].

\section{Acknowledgment}

We would like to thank S. Rouhani for his helpful comments and careful reading of the manuscript.

\section{References}

[1] P. Bak, C. Tang, and K. Wiesenfeld, Phys. Rev. Lett., 59, 381 (1987).

[2] H. J. Jensen, Self-Organized Criticality (Cambridge University Press, Cambridge, 1998).

[3] D. Dhar, cond-mat/9909009].

[4] D. Dhar, Phys. Rev. Lett. 64, 1613 (1990); Phys. Rev. Lett. 64, 2837 (1990).

[5] S. N. Majumdar and D. Dhar, Physica A 185129 (1992).

[6] V. B. Prizzhev, J. Stat. Phys. 74955 (1994).

[7] E. V. Ivashkevich, J. Phys. A. 27. 3643 (1994).

[8] M. Jeng, Phys. Rev. E 71, 016140 (2005) cond-mat/0407115.

[9] M. Jeng, Phys. Rev. E 71, 036153 (2005) cond-mat/0405594.

[10] M. Jeng, Phys. Rev. E 60, 051302 (2004) cond-mat/0312656.

[11] P. Ruelle, Phys. Lett. B 539172 (2002) hep-th/0203105].

[12] P. Ruelle, J. Stat. Mech. (2007) P09013 arXiv:0707.3766

[13] G. Piroux and P. Ruelle, J. Stat. Mech., 0410 (2004) P005, hep-th/0407143.

[14] S. Moghimi-Araghi, M. A. Rajabpur, S. Rouhani, Nucl. Phys. B 718 [FS](2005) 362, cond-mat/0410434.

[15] M. Jeng, G. Piroux, P. Ruelle, J. Stat. Mech. 0610 (2006) P015, cond-mat/0609284.

[16] E. V. Ivashkevich, J. Phys. A 32 1691(1999).

[17] S. Mahieu And P. Ruelle, Phys. Rev. E 64 066130, hep-th/0107150]. 
[18] S. Moghimi-Araghi, A. Nejati, J. Phys. A: Math. Theor. 40 ,2007) 11277, cond-mat/0612224

[19] Phys. Rev. Lett. 63, 470 (1989).

[20] A. Gabrielov, Physica A 195, (1993) 253.

[21] Anne Fey, Ronald Meester, Corrie Quant, Frank Redig, math-ph/0701029].

[22] P. Ghaffari, S. Lise and H. J. Jensen, Phys. Rev. E 566702 (1997).

[23] T. Tsuchia and M. Katori, Phys. Rev. E 611183 (2000).

[24] A. Vazquez, Phys. Rev. E 627797 (2000).

[25] S. S. Manna, L. B. Kiss and J. Kertesz, J. Stat. Phys. 61923 (1990).

[26] H. Widom, Amer. J. Math. 95333 (1973).

[27] N. Azimi-Tafreshi, S. Moghimi-Araghi, in preparation. 